\title{
Rationale, study design and implementation of the COLM study: the combination of OLMesartan and calcium channel blocker or diuretic in high-risk elderly hypertensive patients
}

Toshio Ogihara, Takao Saruta, Hiromi Rakugi, Kazuaki Shimamoto, Sadayoshi Ito, Hiroaki Matsuoka, Masatsugu Horiuchi, Tsutomu Imaizumi, Shuichi Takishita, Jitsuo Higaki, Shigehiro Katayama, Ikuo Saito and Kazuyuki Shimada, on behalf of the COLM study investigators

Hypertension Research (2009) 32, 319; doi:10.1038/hr.2009.35

Correction to: Hypertension Research (2009) 32, 163-167; doi:10.1038/ hr.2008.31

During typesetting and correction of the above paper, incorrect affiliations were applied to the authors of the above paper and published as such. The publishers apologize for this and are now happy to correct this mistake-the correct affiliations are shown below:

Toshio Ogihara ${ }^{1}$, Takao Saruta ${ }^{2}$, Hiromi Rakugi ${ }^{1}$, Kazuaki Shimamoto $^{3}$, Sadayoshi Ito $^{4}$, Hiroaki Matsuoka ${ }^{5}$, Masatsugu Horiuchi ${ }^{6}$, Tsutomu Imaizumi ${ }^{7}$, Shuichi Takishita $^{8}$, Jitsuo Higaki ${ }^{6}$, Shigehiro
Katayama $^{9}$, Ikuo Saito ${ }^{2}$ and Kazuyuki Shimada ${ }^{10}$, on behalf of the COLM study investigators

${ }^{1}$ Osaka University Graduate School of Medicine, Osaka, Japan; ${ }^{2}$ Keio University, Tokyo, Japan; ${ }^{3}$ Sapporo Medical University School of Medicine, Sapporo, Japan; ${ }^{4}$ Tohoku University Graduate School of Medicine, Sendai, Japan; ${ }^{5}$ Dokkyo Medical University, Tochigi, Japan; ${ }^{6}$ Ehime University Graduate School of Medicine, Ehime, Japan; ${ }^{7}$ Kurume University School of Medicine, Kurume, Japan; ${ }^{8}$ University of the Ryukyus School of Medicine, Okinawa, Japan; ${ }^{9}$ Saitama Medical University, Saitama, Japan and ${ }^{10}$ Jichi Medical University, Shimotsuke, Japan 